\title{
A MIXED FINITE ELEMENT METHOD FOR ELASTICITY IN THREE DIMENSIONS
}

\author{
By
}

\section{Scot Adams}

and

Bernardo Cockburn

IMA Preprint Series \# 1983

( July 2004)

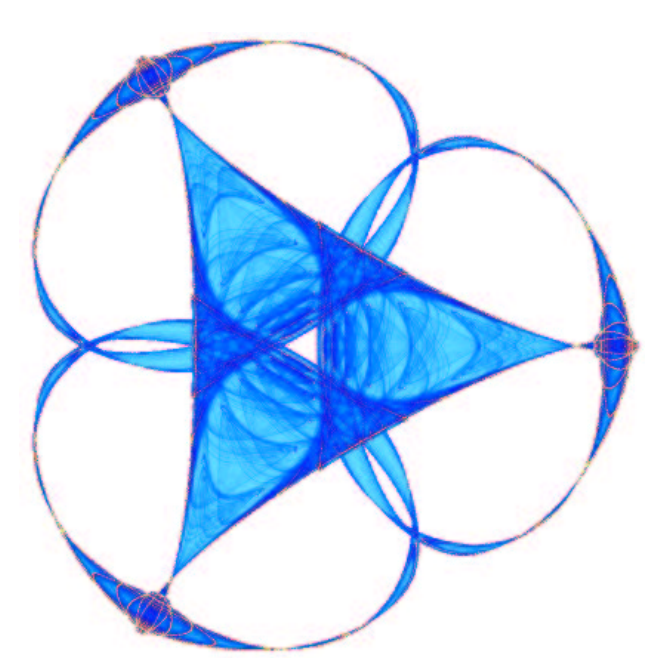

INSTITUTE FOR MATHEMATICS AND ITS APPLICATIONS

UNIVERSITY OF MINNESOTA

514 Vincent Hall

206 Church Street S.E.

Minneapolis, Minnesota 55455-0436

Phone: 612/624-6066 Fax: 612/626-7370

URL: http://www.ima.umn.edu 


\title{
A MIXED FINITE ELEMENT METHOD FOR ELASTICITY IN THREE DIMENSIONS
}

\author{
SCOT ADAMS AND BERNARDO COCKBURN
}

ABSTRACT. We describe a stable mixed finite element method for linear elasticity in three dimensions.

\section{INTRODUCTION}

In the writeup ([Arnold02]) of his plenary address to the 2002 International Congress of Mathematicians, at the end of the third paragraph of Section 6, D. Arnold asserts: "Four decades of searching for mixed finite elements for elasticity, beginning in the 1960s did not yield any stable elements with polynomial shape functions". In [AW92], Arnold and R. Winther give stable elements for a two-dimensional version of problem, but the three-dimensional problem remained open. Here we announce an analogous scheme for three dimensions.

We would like to thank Doug Arnold for numerous detailed conversations and Vic Reiner for developing the Mathematica program described below.

\section{Description of the ELEMENTS}

In what follows, the term "chamber" will mean a codimension-zero simplex, "facet" will mean a codimension-one simplex and "face" will mean a simplex of any codimension. We encourage collaboration with combinatorialists, who generally follow this terminology.

In Section 3 of [AW92], we see an explicit description of a polynomial function space $\Sigma_{T}$, together with 24 degrees of freedom that give a stable finite element space for a two-dimensional version of the mixed elasticity problem. We indicate here the three-dimensional version of $\Sigma_{T}$ and the required unisolvent degrees of freedom.

Date: 15 June $2004 \quad$ Printout date: July 27, 2004.

1991 Mathematics Subject Classification. 74B05, 65N30.

Key words and phrases. finite element methods, elasticity, mixed methods. 
Let $\mathbb{S}$ denote the six-dimensional space of $3 \times 3$ symmetric matrices with real entries. Let $T$ be a tetrahedron in $\mathbb{R}^{3}$. Let $V_{T}$ be the set of $\mathbb{R}^{3}$-valued polynomials on $T$ of degree less than or equal to 1 . Let $\Sigma_{T}$ denote those $\mathbb{S}$-valued polynomials $\sigma$ on $T$ with degree less than or equal to 4 and with the property that $\operatorname{div} \sigma$ has degree less than or equal to 1 . (Here $\operatorname{div} \sigma$ is the row-divergence of $\sigma$, following the notation of [AW92].) Then $\operatorname{dim} \Sigma_{T}=162$. The required 162 degrees of freedom are:

(1) the average of $\sigma$ over $T$ (6 degrees of freedom);

(2) for each facet $f$, with normal $n$, the constant and linear moments over $f$ of $\sigma n(4 \times 3 \times 3=36$ degrees of freedom);

(3) for each edge $e$, parallel to a vector $t$, adjacent to facets $f_{1}$ and $f_{2}$ with normals $n_{1}$ and $n_{2}$, the constant, linear and quadratic moments over $e$ of $\sigma n_{1} \cdot t, \sigma n_{2} \cdot t, \sigma n_{1} \cdot n_{1}, \sigma n_{2} \cdot n_{2}, \sigma n_{1} \cdot n_{2}=\sigma n_{2} \cdot n_{1}$ $(6 \times 3 \times 5=90$ degrees of freedom);

(4) for each edge $e$, parallel to a vector $t$, the average over $e$ of $\sigma t \cdot t$ (6 degrees of freedom); and

(5) the values of $\sigma$ at the vertices of $T(4 \times 6=24$ degrees of freedom).

Note that, for a function $\sigma$, all the above 162 degrees of freedom vanish if and only if the following all vanish:

(1) the average of $\sigma$ over $T$;

(2) for each facet $f$, with normal $n$, the constant and linear moments over $f$ of $\sigma n$;

(3') for each edge $e$, adjacent to facets $f_{1}$ and $f_{2}$ with normals $n_{1}$ and $n_{2}$, the constant, linear and quadratic moments over $e$ of $\sigma n_{1}$ and $\sigma n_{2}$;

(4') for each edge $e$, the average over $e$ of $\sigma$;

(5) the values of $\sigma$ at the vertices of $T$.

The unisolvency of this system was originally verified on a specific tetrahedron via a Mathematica program written by V. Reiner, to whom we owe a debt of gratitude. For the code of this program, please point to:

http://www.math.umn.edu/ adams/elasticity.txt

Note that if unisolvency is verified on one tetrahedron, then it follows on any other tetrahedron by the affine transformation rule of equation (4.4) of [AW92]. (Note: Because of the form of this equation, one 
A MIXED FINITE ELEMENT METHOD FOR ELASTICITY IN THREE DIMENSIONS

should avoid the vector $t$ of (3) and (4) above, instead using (3') and $\left(4^{\prime}\right)$.)

Eventually the second named author found a direct proof of unisolvency that is given in the last section of this paper.

Note that 0 is the only polynomial function on a facet $f$ of $T$ of degree smaller than or equal to 4 , which vanishes at each vertex of $f$, which has vanishing constant\&linear\&quadratic moments along each edge of $f$, and which has vanishing constant\&linear moments along $f$ itself. This ensures that the assembled space is in $H$ (div).

Let $\Omega$ be an open connected polyhedron in $\mathbb{R}^{3}$ with a mesh $M$. Let $\Sigma$ be the assembled space associated to $M$ with the degrees of freedom $(1)-(5)$ listed above. Let $\Pi: H^{1}(\Omega, \mathbb{S}) \rightarrow \Sigma$ be any projection map that preserves all degrees of freedom associated to chambers and facets (i.e., to faces of codimension at most 1). Let $V$ be the full space of all functions on $\Omega$ which are piecewise polynomial, and of degree at most 1 on each chamber. Let $P: L^{2}\left(\Omega, \mathbb{R}^{3}\right) \rightarrow V$ be orthogonal projection. We have div $: H^{1}(\Omega, \mathbb{S}) \rightarrow L^{2}\left(\Omega, \mathbb{R}^{3}\right)$ and div $: \Sigma \rightarrow V$. Equation (3.6) of [AW92] asserts that $\operatorname{div} \circ \Pi=P \circ$ div. It is proved in the three-dimensional case without any significant change.

Thus the results and statements in Section 3 of [AW92] go through in a straightforward way in three dimensions. Note that the six degrees of freedom described in (4) above are not used, except in the proof of unisolvency. There are undoubtedly possible replacements for those six. More generally, we expect there are numerous other solutions to the stated problem.

We have thus far not attempted to analyze the other sections of [AW92], but hope that much will proceed in the three-dimensional case in a similar way to what was done in the two-dimensional case.

\section{UNISOLVENCY OF THE SYSTEM}

Let $T$ be a tetrahedron in $\mathbb{R}^{3}$, i.e., the convex hull of four points not lying on a plane. Let $\sigma \in \Sigma_{T}$ and assume that the degrees of freedom described in (1)-(5) all vanish. We are going to show that $\sigma=0$.

To do that, we begin by listing the properties we are going to use to prove this result. They are the following:

(6) $\int_{T} \sigma=0$;

(7) $\sum_{j} \sigma_{i j, j}=0$ on $T$; 
(8) $\sum_{j} \sigma_{i j} n_{j}=0$ on $\partial T$;

(9) for any edge $e$ in $T, \int_{e} \sigma=0$; and

(10) $\sigma_{i j}=\sigma_{j i}$.

The properties (6) and (7) follow as in the proof of Lemma 3.1 of [AW92].

Next, we introduce the notation needed for the proof. Let $\left\{x_{i}\right\}_{i=1}^{4}$ be the set of vertices of $T$. For all integers $l \in[1,4]$, let $\bar{n}_{l}$ be the outward pointing normal to the facet containing $\left\{x_{i}\right\}_{i \neq l}$, and let $h_{l}$ be the distance from $x_{l}$ to that plane.

For all integers $k \geq 0$, let $P^{k}$ denote the vector space of all polynomials $\mathbb{R}^{3} \rightarrow \mathbb{R}$ such that the total degree of any term is at most $k$. For any integer $l \in[1,4]$, we denote by $\lambda_{l} \in P^{1}$ the affine function such that $\lambda_{l}\left(x_{i}\right)=\delta_{l i}$, where $\delta$ is the Kronecker delta. Set

(11) $\bar{g}_{l}:=\nabla \lambda_{l}=-\left(1 / h_{l}\right) \bar{n}_{l}$.

Then, as $\nabla\left(\sum_{l=1}^{4} \lambda_{l}\right)=\nabla 1=\overline{0}$, we get

(12) $\sum_{l=1}^{4} \bar{g}_{l}=\overline{0}$.

We are now ready to prove the unisolvency of the system. We proceed in several steps.

Claim a: For all integers $l, m \in[1,4]$, there exists $\phi_{l m} \in P^{2}$ such that $\bar{g}_{m} \sigma \bar{g}_{l}=\lambda_{l} \lambda_{m} \phi_{l m}$.

Proof: By (11), we have $\sigma \bar{g}_{l}=-\left(1 / h_{l}\right) \sigma \bar{n}_{l}$. Then, by (8), we get $\sigma \bar{g}_{l}=0$ on $\left\{\lambda_{l}=0\right\}$. It follows that $\sigma \bar{g}_{l}$ is divisible by $\lambda_{l}$ in $P^{4} \times P^{4} \times P^{4}$. Then $\bar{g}_{m} \sigma \bar{g}_{l}$ is divisible by $\lambda_{l}$ in $P^{4}$. Similarly, $\bar{g}_{m} \sigma \bar{g}_{l}$ is divisible by $\lambda_{m}$ in $P^{4}$, so we are done if $l \neq m$.

We therefore assume $l=m$. We already know that $\sigma \bar{g}_{l}=\lambda_{l} \bar{\phi}_{l}$ for some $\bar{\phi}_{l}$ in $P^{3} \times P^{3} \times P^{3}$. By (7) and (10), $0=\nabla \cdot\left(\sigma \bar{g}_{l}\right)=\nabla \cdot\left(\lambda_{l} \bar{\phi}_{l}\right)$, and, since $\nabla \lambda_{l}=\bar{g}_{l}$, this leads to $0=\bar{g}_{l} \cdot \bar{\phi}_{l}+\lambda_{l} \nabla \cdot \bar{\phi}_{l}$. Then

$$
\bar{g}_{l} \sigma \bar{g}_{l}=\lambda_{l} \bar{\phi}_{l} \cdot \bar{g}_{l}=-\lambda_{l}^{2} \nabla \cdot \bar{\phi}_{l},
$$

and, since $\nabla \cdot \bar{\phi}_{l} \in P^{2}$, the result follows. End of proof of Claim a.

Claim b: For any edge e, we have $\sigma=0$ on e. Proof: Let us proof the result for the edge $e=\left\{\lambda_{3}=\lambda_{4}=0\right\}$, the proof on the other edges being similar. It is enough to show that $\bar{g}_{m} \sigma \bar{g}_{l}=0$ on $e$ for all integers $l, m \in[1,4]$. By Claim a, we are done if $l \in[3,4]$ or if $m \in[3,4]$. We therefore assume that $l, m \in[1,2]$.

By (12) and Claim a, we see that, on the edge $e$,

$$
\bar{g}_{1} \sigma \bar{g}_{1}=\bar{g}_{1} \sigma\left(-\bar{g}_{2}-\bar{g}_{3}-\bar{g}_{4}\right)=-\bar{g}_{1} \sigma \bar{g}_{2} \text {. }
$$


A MIXED FINITE ELEMENT METHOD FOR ELASTICITY IN THREE DIMENSIONS

By (12) and Claim a again, we have

$$
-\bar{g}_{1} \sigma \bar{g}_{2}=\left(\bar{g}_{2}+\bar{g}_{3}+\bar{g}_{4}\right) \sigma \bar{g}_{2}=\bar{g}_{2} \sigma \bar{g}_{2} \text {. }
$$

Then, on $e$, we get $\bar{g}_{1} \sigma \bar{g}_{1}=-\bar{g}_{1} \sigma \bar{g}_{2}=\bar{g}_{2} \sigma \bar{g}_{2}$. Then, since by Claim a, $\bar{g}_{1} \sigma \bar{g}_{1}$ is divisible by $\lambda_{1}^{2}$ in $P^{4}$ and $\bar{g}_{2} \sigma \bar{g}_{2}$ by $\lambda_{2}^{2}$ in $P^{4}$, we have that, for some constant $c$, we can write

$$
\bar{g}_{1} \sigma \bar{g}_{1}=\bar{g}_{2} \sigma \bar{g}_{2}=c \lambda_{1}^{2} \lambda_{2}^{2} .
$$

Since, by (9), $\int_{e} \sigma=0$, we conclude that $c=0$, and so

$$
\bar{g}_{1} \sigma \bar{g}_{1}=-\bar{g}_{1} \sigma \bar{g}_{2}=\bar{g}_{2} \sigma \bar{g}_{2}=0,
$$

as desired. End of proof of Claim b.

Claim c: For any integers $l, m \in[1,4]$, we have $\int_{T} \lambda_{m} \bar{g}_{l} \sigma \bar{g}_{l}=0$. Proof: Let us prove the result for $l=1$, the proof in general being similar. By (7) and (8), we have

$$
\int_{T} \sum_{i, j} \sigma_{i j} v_{i, j}=-\left[\int_{T} \sum_{i, j} \sigma_{i j, j} v_{i}\right]+\left[\int_{\partial T} \sum_{i, j} \sigma_{i j} n_{j} v_{i}\right]=0 .
$$

For $v_{i}=a_{i} \lambda_{1}^{\alpha} \lambda_{m}^{\beta}$, we have

$$
v_{i, j}=a_{i} \alpha \lambda_{i}^{\alpha-1} \lambda_{m}^{\beta}\left(g_{1}\right)_{j}+a_{i} \beta \lambda_{i}^{\alpha} \lambda_{m}^{\beta-1}\left(g_{m}\right)_{j},
$$

and so

(13) $0=\int_{T} \alpha \lambda_{1}^{\alpha-1} \lambda_{m}^{\beta} \bar{a} \sigma \bar{g}_{1}+\int_{T} \beta \lambda_{1}^{\alpha} \lambda_{m}^{\beta-1} \bar{a} \sigma \bar{g}_{m}$.

For $\beta=0, \alpha-1=\gamma, \bar{a}=\bar{g}_{m}$, (13) yields

(14) $0=\int_{T} \lambda_{1}^{\gamma} \bar{g}_{m} \sigma \bar{g}_{1}$,

and, for $\alpha=1, \beta=1$ and $\bar{a}=\bar{g}_{1}$, (13) yields

$$
0=\left[\int_{T} \lambda_{m} \bar{g}_{1} \sigma \bar{g}_{1}\right]+\left[\int_{T} \lambda_{1} \bar{g}_{1} \sigma \bar{g}_{m}\right] .
$$

Using (14) with $\gamma=1$ and the symmetry (see (10)) of $\sigma$, we see that $\int_{T} \lambda_{1} \bar{g}_{1} \sigma \bar{g}_{m}=0$, and the result follows. End of proof of Claim $c$.

Claim d: For all integers $l \in[1,4]$, we have $\bar{g}_{l} \sigma \bar{g}_{l}=0$ on T. Proof: Let us prove the result for $l=1$, the proof in general being similar.

Since by Claim $a, \bar{g}_{1} \sigma \bar{g}_{1}=\lambda_{1}^{2} \phi_{11}$, we get

$$
\phi_{11}=\lambda_{2} c_{3} \lambda_{4}+\lambda_{2} \lambda_{3} c_{4}+c_{2} \lambda_{3} \lambda_{4},
$$

by Claim b. Hence

(15) $\bar{g}_{1} \sigma \bar{g}_{1}=\lambda_{1}^{2}\left(\lambda_{2} c_{3} \lambda_{4}+\lambda_{2} \lambda_{3} c_{4}+c_{2} \lambda_{3} \lambda_{4}\right)$ 
From Claim $c$ and (15), we see, for all integers $m \in[1,4]$, that

$$
0=\int_{T} \lambda_{m} \lambda_{1}^{2}\left(\lambda_{2} c_{3} \lambda_{4}+\lambda_{2} \lambda_{3} c_{4}+c_{2} \lambda_{3} \lambda_{4}\right) .
$$

Now, for all integers $\alpha_{1}, \alpha_{2}, \alpha_{3}, \alpha_{4} \geq 0$, we have

$$
\frac{1}{|T|} \int_{T} \lambda_{1}^{\alpha_{1}} \lambda_{2}^{\alpha_{2}} \lambda_{3}^{\alpha_{3}} \lambda_{4}^{\alpha_{4}}=\frac{\alpha_{1} ! \alpha_{2} ! \alpha_{3} ! \alpha_{4} ! 3 !}{\left(\alpha_{1}+\alpha_{2}+\alpha_{3}+\alpha_{4}+3\right) !},
$$

where $|T|$ is the volume of the tetrahedron. A simple computation shows then that $c_{2}=c_{3}=c_{4}=0$. This implies that $\bar{g}_{1} \sigma \bar{g}_{1}=0$ on $T$. End of proof of Claim d.

Claim e: $\sigma=0$ on T. Proof: Let $l, m \in[1,4]$ be integers. We wish to show that $\bar{g}_{m} \sigma \bar{g}_{l}=0$ on $T$. By Claim $d$, we may assume that $l \neq m$. We take $l=1$ and $m=2$, the proof in general being similar.

By Claim $d$ and the symmetry of $\sigma,(10)$, we have that

$$
\bar{g}_{1} \sigma \bar{g}_{2}=\frac{1}{2}\left(\bar{g}_{1}+\bar{g}_{2}\right) \sigma\left(\bar{g}_{1}+\bar{g}_{2}\right) \text {. }
$$

Since, by (12), $\bar{g}_{1}+\bar{g}_{2}=-\bar{g}_{3}-\bar{g}_{4}$, we get

$$
\bar{g}_{1} \sigma \bar{g}_{2}=\frac{1}{2}\left(\bar{g}_{3}+\bar{g}_{4}\right) \sigma\left(\bar{g}_{3}+\bar{g}_{4}\right)=\bar{g}_{3} \sigma \bar{g}_{4}
$$

again by Claim $d$ and (10). This implies, by Claim a, that $\bar{g}_{1} \sigma \bar{g}_{2}$ is divisible both by $\lambda_{1} \lambda_{2}$ and by $\lambda_{3} \lambda_{4}$ in $P^{4}$. As a consequence, there is a constant $c$ for which we have $\bar{g}_{1} \sigma \bar{g}_{2}=\lambda_{1} \lambda_{2} \lambda_{3} \lambda_{4} c$. Then (6) yields $c=0$, so $\bar{g}_{1} \sigma \bar{g}_{2}=0$, as desired. End of proof of Claim e.

This completes the proof of the unisolvency of the system.

\section{REFERENCES}

[Arnold02] D. Arnold: Differential complexes and numerical stability. In: Proceedings of the International Congress of Mathematicians, Vol. I (Beijing, 2002), 137-157, Higher Ed. Press, Beijing, 2002.

[AW92] D. Arnold and R. Winther: Mixed finite elements for elasticity Numer. Math. 92 (2002):401-419.

School of Mathematics, University of Minnesota, Minneapolis, MN 55455,ADAMS@MATH.UMN.EDU AND COCKBURN@MATH.UMN.EDU 